UVX 2012, 01008 (2013)

DOI: $10.1051 / \mathrm{uvx} / 201301008$

(C) Owned by the authors, published by EDP Sciences, 2013

\title{
L'étude du Soleil et l'optique X/UV : 50 ans de succès
}

\author{
F. Auchère ${ }^{1}$ et F. Delmotte ${ }^{2}$ \\ 1 Institut d'Astrophysique Spatiale, Bâtiment 121, CNRS, Univ. Paris Sud 11, 91405 Orsay, \\ France \\ ${ }^{2}$ Laboratoire Charles Fabry, Institut d'Optique, CNRS, Univ. Paris Sud 11, 2 avenue Augustin \\ Fresnel, 91127 Palaiseau Cedex, France
}

\begin{abstract}
Résumé. Depuis les premières expériences embarquées à bord de fusées sondes jusqu'aux satellites modernes d'aujourd'hui, la compréhension de la physique de notre étoile a beaucoup bénéficié des progrès de l'optique X/UV. En effet ce domaine permet de sonder les couches de l'atmosphère solaire où les processus de transfert d'énergie responsables des diverses formes d'activité ont lieu. Nous effectuons une brève revue de cet héritage, en nous concentrant sur l'apparition des optiques multicouches. Nous présentons ensuite les développements technologiques effectués actuellement pour les futurs instruments spatiaux d'observation du Soleil, et en particulier les couches minces EUV développées pour la mission Solar Orbiter de l'Agence Spatiale Européenne qui sera lancée en 2017.
\end{abstract}

\section{POURQUOI OBSERVER LE SOLEIL EN X/UV ?}

La couronne du Soleil est le siège de mécanismes toujours mal compris qui accélèrent le plasma pour former le vent solaire qui se propage dans l'héliosphère à vitesse supersonique. À l'intérieur de l'étoile, le champ magnétique crée par des effets dynamo est transporté vers la surface où il forme de larges boucles qui sont ensuite étirées et cisaillées par la rotation différentielle de l'étoile, accumulant ainsi de grandes quantités d'énergie. Il peut alors se produire des éruptions qui éjectent ces structures magnétiques et le plasma associé à travers le système solaire, provoquant éventuellement des collisions avec le bouclier magnétique de la Terre. Comprendre les processus physiques à l'œuvre dans ce système complexe est le but de ce que l'on appelle maintenant l'héliophysique. Le Soleil étant typique d'étoiles peu massives, ce domaine de recherche concerne l'astrophysique en général, mais est unique car le Soleil est la seule étoile suffisamment proche pour être étudiée avec tout le détail nécessaire.

Il n'existe pas un domaine de longueur d'onde ni une technique d'observation privilégié pour l'étude du Soleil. Les domaines spectraux étudiés vont des gammas aux ondes radio, le choix de telle ou telle gamme de longueur d'onde dépend des phénomènes physiques et/ou des couches de l'étoile que l'on désire sonder. Le domaine X/UV est particulièrement adapté à l'étude aux couronnes du Soleil et des étoiles, c'est-à-dire leurs enveloppes les plus externes. En effet, si la couronne est bien visible en lumière blanche (c'est elle qui est révélée durant les éclipses totales), du fait des températures de plusieurs million de degrés atteintes par les plasmas coronaux, la plupart des raies spectrales sont émises à plus courtes longueurs d'onde. On voit ainsi dans le spectre de la Figure 1 obtenu le 4 avril 1969 avec un spectromètre en incidence rasante par Malinovsky \& Heroux [1] de nombreuses raies émises par des ions de degrés d'ionisation élevés formés à des températures comprises entre $3 \times 10^{5} \mathrm{~K}(\mathrm{O} \mathrm{v})$ et 2,5 × $10^{6} \mathrm{~K}$ (Ni XIV).

This is an Open Access article distributed under the terms of the Creative Commons Attribution License 2.0, which permits unrestricted use, distribution, and reproduction in any medium, provided the original work is properly cited. 


\section{Web of Conferences}

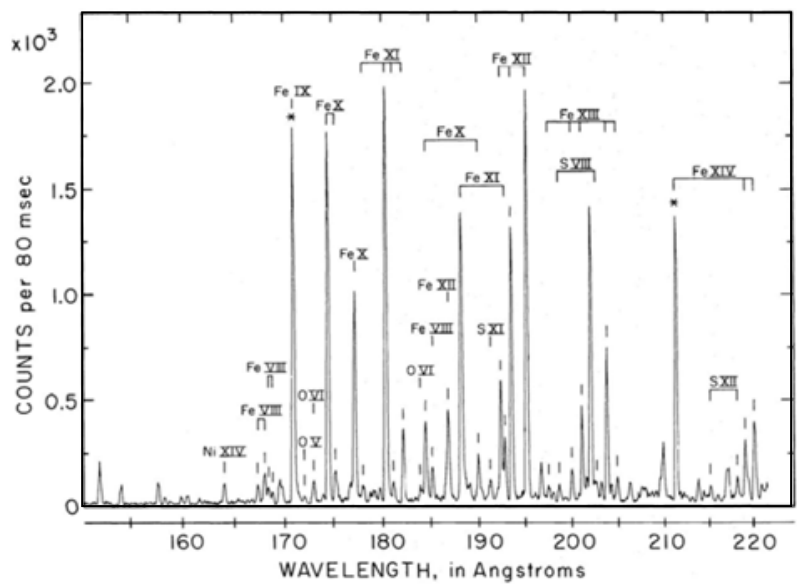

Figure 1. Spectre du Soleil entier obtenu en 1969 [1]. Les degrés d'ionisation élevés témoignent des températures atteintes dans la couronne solaire. Nombre de raies d'émission de ces ions se trouvent dans le domaine X/UV.

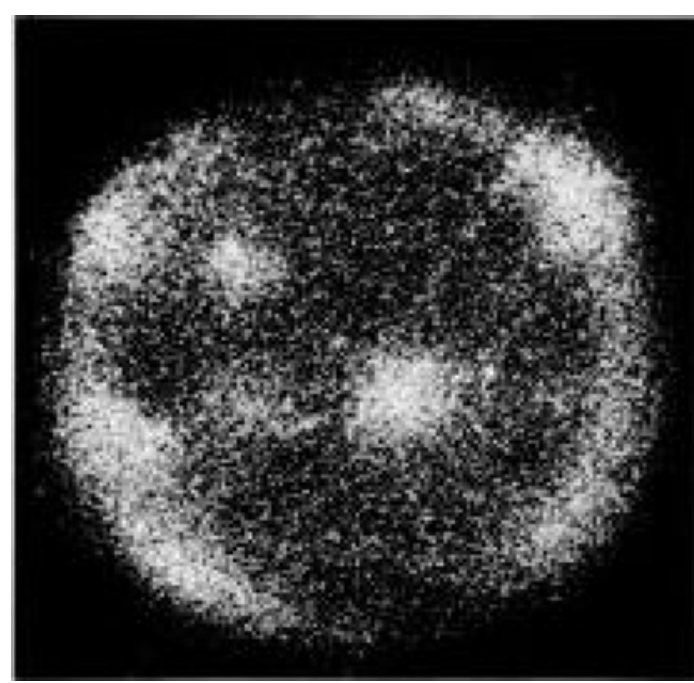

Figure 2. Une des premières images du Soleil obtenue dans de domaine X (20-60 Å, 1965).

\section{UN BREF HISTORIQUE}

Je présente ci-dessous un rapide historique qui n'a pas la prétention d'être exhaustif mais qui résume les grandes évolutions ayant mené aux optiques multicouches modernes.

\subsection{Les années 60 et 70}

Le Soleil a été l'un des premiers objets astrophysiques étudiés dans les nouvelles fenêtres spectrales ouvertes par l'accès à l'espace. Les émissions X du Soleil ont commencé à être explorées à la fin des années 50 et au début des années 60 avec des instruments embarqués à bord de fusées sondes. La première image obtenue le 19 avril 1960 dans le domaine X (20-60 Å) [2,3] était entachée d'un fort roulis de la fusée mais rapidement, des images stabilisées ont pu être obtenues (Figure 2, [4,5]). 


\section{UVX 2012}

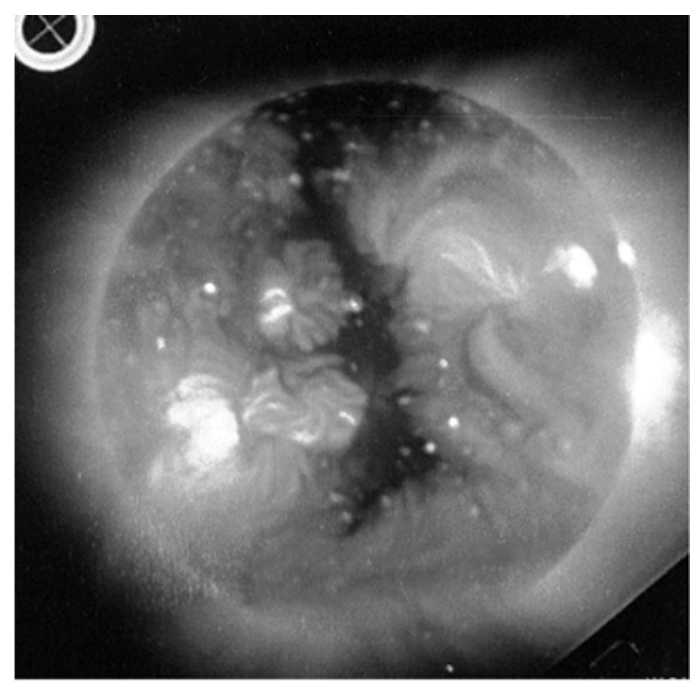

Figure 3. Image du Soleil obtenue par le télescope X de la station spatiale Skylab le 31 mai 1973.

Ces images, obtenues avec des caméras à sténopé, avaient des résolutions spatiales faibles (quelques minutes d'arc) comparativement aux standards actuels (quelques dixièmes de secondes d'arc), mais montraient déjà les principaux types de structures typiques de l'aspect du Soleil à ces longueurs d'onde. On distingue clairement plusieurs régions actives, le fort embrillancement au bord, et les régions polaires sombres connues aujourd'hui sous le nom de trous coronaux.

Après cette époque d'exploration, la série des satellites Orbiting Solar Observatories (OSO 1 à 8) lancés entre 1962 et 1975, puis les missions Skylab en 1973 et 1974 ont permis d'obtenir des spectres et des images X/UV de grande qualité. L'image de la Figure 3, enregistrée le 31 mai 1973 par l'un des télescopes de Skylab [6], montre le gain de résolution remarquable obtenu en seulement 8 ans (cf. Figure 2). Skylab emportait aussi, entre autres, un spectrohéliographe fournissant des superpositions d'images monochromatiques entre 171 et $630 \AA$ [7]. Les instruments des OSOs et de Skylab ont mené à des avancées majeures de notre compréhension de la couronne solaire et certaines de leurs observations font toujours référence.

\subsection{Les années 80 et 90 : apparition et développement des optiques multicouches}

Jusque dans les années 80, la plupart des images du Soleil dans le domaine X ou EUV ont été obtenue par des télescopes de Wolter fonctionnant en incidence rasante. Mais les développements des optiques multicouches fonctionnant en incidence normale ont changé la donne en permettant une meilleure résolution, une plus grande efficacité, des bandes passantes plus étroites, et des niveaux de lumière diffusée plus faibles. La première image du Soleil utilisant cette nouvelle technologie a été obtenue en 1985 par un télescope embarqué à bord d'un fusée sonde. La bande passante du revêtement multicouche W/C était centrée une raie du Si XII à $44 \AA$ [8]. Des images d'une résolution d'environ 1 second d'arc ont rapidement pu être obtenues avec ce type d'instrument. La Figure 4 montre une image obtenue en 1987 par A. Walker et al. [9] avec des multicouches Mo/Si centrées sur 171 A (Fe x) réalisées bar T. Barbee du Lawrence Livermore National Laboratory (LLNL).

Les avantages indéniables de cette technique l'ont fait se développer rapidement. Son utilisation s'est répandue aux spectrographes, avec la première utilisation d'un réseau recouvert de multicouches pour le vol suborbital de 1991 du spectrographe EUV SERTS [10]. Le revêtement Ir/Si réalisé à GSFC par R. Keski-Kuha permettait un gain d'efficacité d'un facteur 9 autour de $300 \AA$ par rapport à un dépôt d'or 


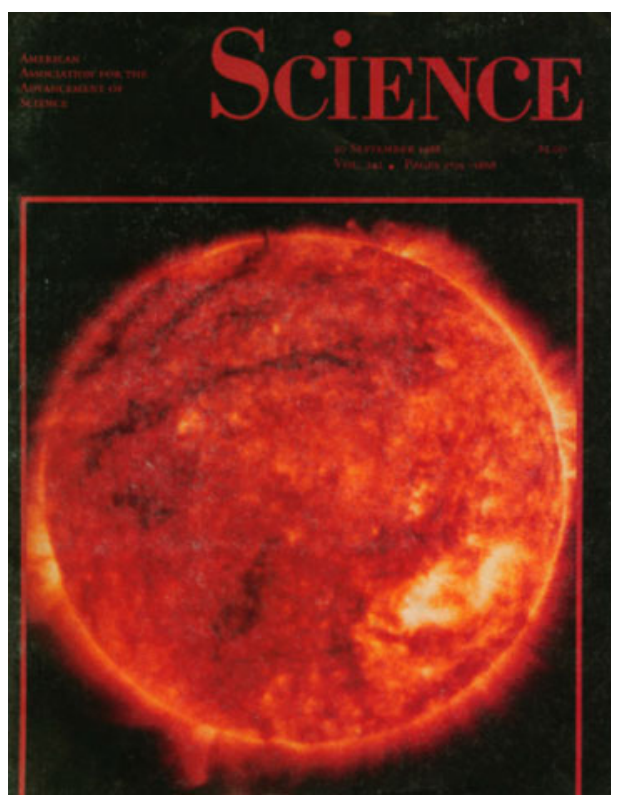

Figure 4. Image monochromatique du Soleil obtenue en 1987 avec un télescope en incidence normale utilisant des multicouches Mo/Si formant une bande passante étroite centrée sur une raie du Fe X à $171 \AA$ A.

Tableau 1. Instruments d'observation du Soleil utilisant des multicouches depuis 1990.

\begin{tabular}{|c|c|c|c|c|c|}
\hline Instr/Mission & Type & Bande $(\mathbf{\AA})$ & Matériaux & Source & Période \\
\hline SERTS/Fusée sonde & Spectrographe & $300-335$ & & GSFC & $1991-93$ \\
\hline EIT/SOHO & Imageur & $171 / 195 / 284 / 304$ & $\mathrm{Mo} / \mathrm{Si}$ & IO & $96-$ \\
\hline TRACE & Imageur & $171 / 195 / 284$ & $\mathrm{Mo} / \mathrm{Si}$ & $\mathrm{LLNL}$ & $98-10$ \\
\hline SPIRIT/CORONAS-F & Imageur & $171 / 195 / 284 / 304$ & $\mathrm{Mo} / \mathrm{Si}$ & $\mathrm{IO}$ & $2001-05$ \\
\hline EUVI/STEREO & Imageur & $171 / 195 / 284 / 304$ & $\mathrm{Mo} / \mathrm{Si}$ & $\mathrm{IO}$ & $2006-$ \\
\hline MOSES/Fusée sonde & Spectro-imageur & 304 & $\mathrm{~B} 4 \mathrm{C} / \mathrm{Mg}_{2} \mathrm{Si}$ & $\mathrm{GSFC}$ & 2006 \\
\hline EIS/Hinode & EIS/Hinode & $170-210 / 250-290$ & $\mathrm{Mo} / \mathrm{Si}$ & $\mathrm{RXO}$ & $2006-$ \\
\hline TESIS/CORONAS-Photon & Imageur & $131 / 304$ & & $\mathrm{IPM}$ & 2009 \\
\hline HECOR/Fusée sonde & Coronographe & 304 & $\mathrm{Mo} / \mathrm{Si} / \mathrm{B} 4 \mathrm{C}$ & $\mathrm{IO}$ & 2009 \\
\hline & & 94 & $\mathrm{Mo} / \mathrm{Y}$, & & \\
AIA/SDO & Imageur & $131 / 171 / 193 / 211$ & $\mathrm{Mo} / \mathrm{Si}$, & $\mathrm{RXO}$ & \\
& & $304 / 335$ & $\mathrm{SiC} / \mathrm{Si}$ & $\mathrm{LLNL}$ & $2010-$ \\
\hline SWAP/Proba 2 & Imageur & 171 & $\mathrm{Mo} / \mathrm{Si}$ & $\mathrm{IO}$ & $2010-$ \\
\hline HiC/Fusée sonde & Imageur & 171 & $?$ & $\mathrm{RXO}$ & 2012 \\
\hline EUI/Solar Orbiter & Imageur & $171 / 304$ & $\mathrm{Al} / \mathrm{Mo} / \mathrm{SiC}$ & $\mathrm{IO}$ & 2017 \\
\hline METIS/Solar Orbiter & Spectro-imageur & 304 & $\mathrm{Mo} / \mathrm{Si}$ & $\mathrm{RXO}$ & 2017 \\
\hline
\end{tabular}

standard. La maturité est atteinte avec l'instrument EIT de la sonde SOHO [11] qui observe le Soleil en continu depuis le 2 janvier 1996 dans quatre bandes du spectre EUV. Les multicouches Mo/Si réalisées par J.-P. Chauvineau à l'Institut d'Optique [12] centrées sur 171 (Fe X), 195 (Fe XII), 284 (Fe Xv) et $304 \AA$ (He II) font suite au travail préparatoire de l'expérience FRECOPA ayant volé dans les années 80 à bord du satellite LDEF. Le succès remarquable d'EIT s'est traduit depuis par le développement du 


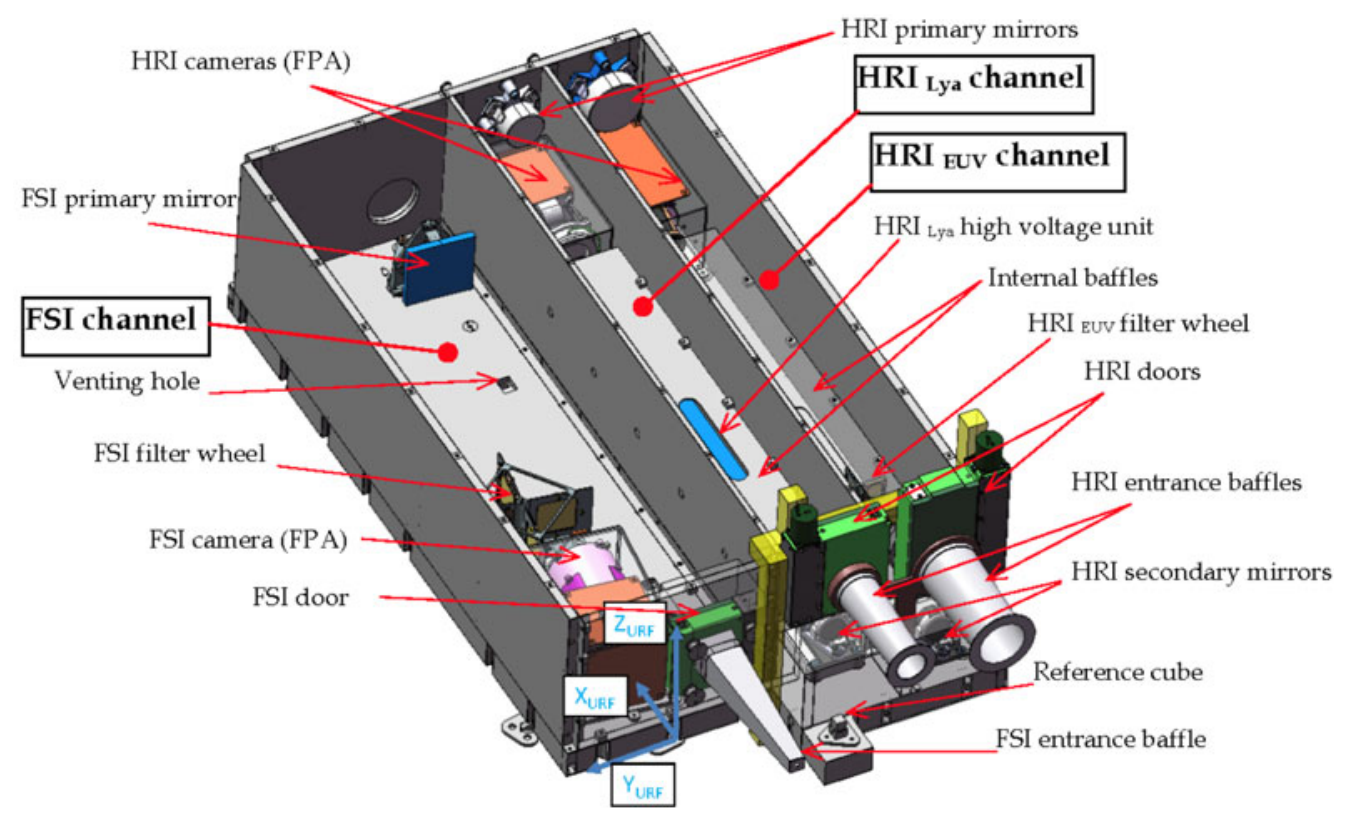

Figure 5. CAO de I'instrument EUI de la mission Solar Orbiter.

même concept instrumental dans de nombreux télescopes (e.g. TRACE [13], SPIRIT [14], EUVI [15], AIA [16]).

La tableau 1 donne un bilan (non exhaustif) d'instruments d'observation du Soleil utilisant des multicouches ayant volé depuis 1990 ou dont le lancement est décidé. Cette technologie est maintenant utilisée aussi bien pour des imageurs que pour des spectrographes ou des coronographes. Après l'utilisation extensive de $\mathrm{Mo} / \mathrm{Si}$, matériaux qui ont prouvé leur résistance à l'environnement spatial, de nouvelles combinaisons sont étudiées aussi bien pour augmenter la réflectivité et/ou la sélectivité que pour étendre la gamme de longueurs d'ondes couvertes. On voit aussi qu'il existe essentiellement quatre groupes réalisant des optiques spatiales : Reflective X-ray Optics et LLNL aux Etats-Unis, l'Institut d'Optique (IO) en France, et l'Institute for Physics of Microstructures (IPM) en Russie.

\section{SOLAR ORBITER}

Le 4 octobre 2011, le Comité des Programmes Scientifiques (SPC) de l'Agence Spatiale Européenne (ESA) approuvé la mission Solar Orbiter pour un lancement en 2017. Elle emportera une charge utile de dix instruments dédiés à l'étude du Soleil et de l'héliosphère, la bulle creusée par la pression du vent solaire dans le milieu interstellaire local. L'orbite de la sonde a été spécialement conçue pour permettre pour la première fois d'amener une batterie complète d'instruments in situ et de télédétection proche du Soleil (0.28 Unité Astronomique) et hors du plan de l'écliptique. En plus de vues à haute résolution (environ $100 \mathrm{~km}$ sur le Soleil) et la possibilité d'observer les pôles (jusqu'à $30^{\circ}$ d'inclinaison par rapport à l'équateur solaire), cette orbite réduit la rotation apparente du Soleil durant les passages au périhélie, permettant ainsi des observations prolongées des régions sources qui produisent les perturbations mesurées in situ dans le vent solaire. Solar Orbiter est une mission très ambitieuse non seulement scientifiquement, mais aussi techniquement. Car la sonde et son instrumentation seront soumises à un environnement très rude nécessitant des solutions spécifiques.

Quatre des cinq instruments de télédétection fonctionnent dans le domaine X/UV (EUI, SPICE, STIX et METIS). Nous nous concentrerons sur EUI (Exteme Ultraviolet Imager, Figure 5, [17]) pour 


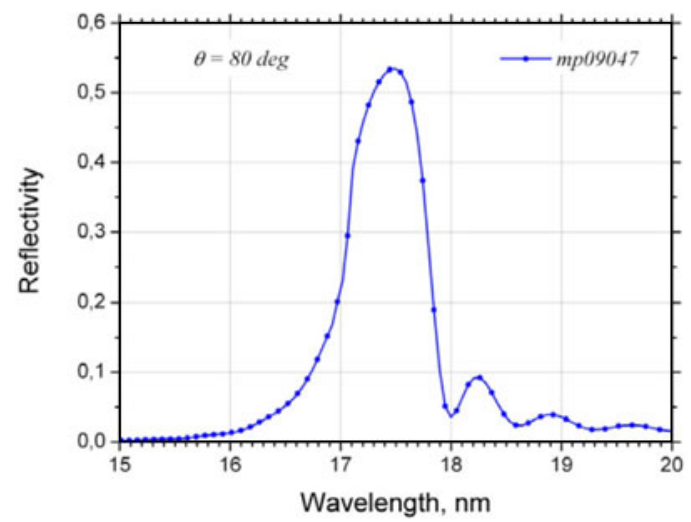

Figure 6. Réflectivité d'une multicouche $\mathrm{Al} / \mathrm{Mo} / \mathrm{SiC}$ optimisée pour $174 \AA$ A.

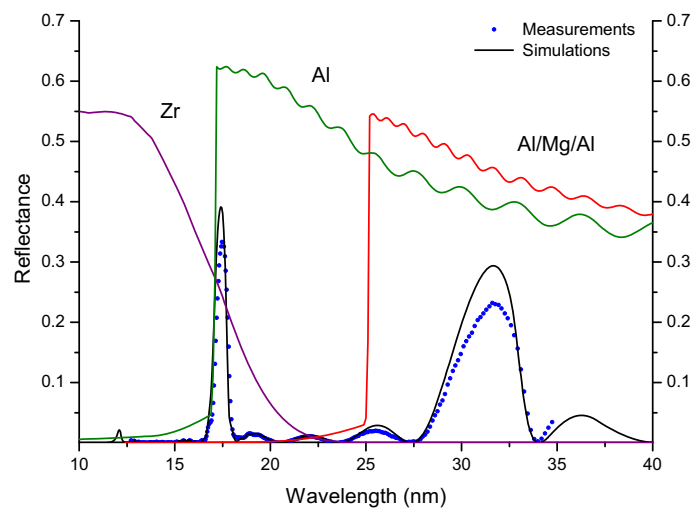

Figure 7. Réflectivité d'une multicouche double bande 174/304 Å, et transmission des filtres utilisés pour isoler les bandes.

lequel l'IO et l'IAS développent des optiques. EUI se compose de deux imageurs à haute résolution HRI (High Resolution Imager) observant à $174 \AA$ (Fe x) et $1216 \AA$ (H I Lyman $\alpha$ ) et d'un imageur à grand champ FSI (Full Sun Imager) qui fonctionne à $174 \AA$ et $304 \AA$ (He II). Le champ d'observation de HRI est de 16,6 minutes d'arc et la résolution spatiale de 1 seconde d'arc. Le champ d'observation de FSI est de 5 degrés avec une résolution spatiale de 4,5 secondes d'arc.

Pour HRI et FSI, nous développons des multicouches $\mathrm{Al} / \mathrm{Mo} / \mathrm{B} 4 \mathrm{C}$ et $\mathrm{Al} / \mathrm{Mo} / \mathrm{SiC}$ qui permettent des gains de réflectivité importants par rapport à la combinaison $\mathrm{Mo} / \mathrm{Si}$, environ un facteur 2 sur un système à deux miroirs (Figure 6, [18,19]). FSI utilise un concept novateur: celui des multicouches double-bande [20]. Ces dépôts sont constitués d'un empilement de deux multicouches séparées par une couche d'espacement. La multicouche de base est une structure classique dont on utilise les premier et deuxième ordres. La multicouche supérieure est constituée de quelques périodes et permet de moduler la réflectivité de la multicouche inférieure. En jouant sur les paramètres de la multicouche supérieure et l'épaisseur de la couche d'espacement, on peut modifier la position des pics ainsi que le nombre et la position des extinctions crées par les interférences entre les deux structures [20,21]. La Figure 7 montre la réflectivité d'un dépôt 171/304 Å développé pour FSI. Le filtre Aluminium situé à l'entrée de l'instrument laisse passer les deux longueurs d'onde. Au plan focal, une roue porte des filtres $\mathrm{Al} / \mathrm{Mg}$ et $\mathrm{Al} / \mathrm{Zr}$ permettant d'isoler l'une ou l'autre des deux bandes. Ce design particulier permet un télescope 


\section{UVX 2012}

à deux canaux mais n'utilisant qu'un seule pupille, ce qui permet de minimiser à la fois le volume, la masse et la température de l'instrument.

\section{PERSPECTIVES}

Les travaux récents, par exemple ceux effectués pour Solar Orbiter, montrent que des progrès significatifs peuvent encore être attendus des optiques multicouches développées pour l'astrophysique, que ce soit par l'utilisation de nouveaux matériaux ou de structures complexes. En plus de gains de réflectivité et d'une plus grande flexibilité dans la forme des bandes passantes obtenues, il existe un besoin net d'étendre la gamme de longueurs d'onde utilisables, en particulier entre $40 \AA$ à $1000 \AA$ où les efficacités des optiques est pour l'instant faible. Mais les développements nécessaires ne se limitent pas aux quelques thématiques évoquées ici. Par exemple, des polariseurs efficaces en UV/EUV permettraient d'avoir accès au champ magnétique dans la couronne du Soleil. Or bien que cette quantité soit particulièrement importante pour la compréhension de la physique de l'étoile, elle est aujourd'hui très difficile à mesurer. Ainsi, tout comme les développements des optiques et des détecteurs X/UV ont nourri la recherche en astrophysique, les développements technologiques à venir seront eux aussi porteurs de nouvelles découvertes.

\section{Références}

[1] M. Malinovsky, L. Heroux, Astrophys. Journ., 181, 1009 (1973)

[2] T.A. Chubb, H. Friedman, R.W. Kreplin, Journ. Geophys. Res. 65, 1831 (1960)

[3] R.L. Blake, T.A. Chubb, H. Friedman, A.E. Unzicker, Astrophys. Journ., 137, 3 (1963)

[4] P.C. Russell, Nature, 205, 684 (1965)

[5] P.C. Russell \& K.A. Pounds, Nature, 209, 490 (1966)

[6] J.H. Underwood, J.E. Milligan, A.C. Deloach \& R.B. Hoover, Appl. Optics, 16, 858 (1977)

[7] R. Tousey et al., Sol. Phys. 33, 265 (1973)

[8] J.H. Underwood et al., Science, 238(4823), 61 (1987)

[9] A.B.C. Walker et al., Science, 241(4874), 1781 (1988)

[10] W.M. Neupert, G.L. Epstein \& R.J. Thomas, Sol. Phys., 137, 87 (1992)

[11] J.-P. Delaboudinière et al., Sol. Phys. 162, 291 (1995)

[12] J.P. Chauvineau et al., Proc. SPIE, 1546, 576 (1991)

[13] B.N. Handy, et al., Sol. Phys., 187, 229 (1999)

[14] I. Zhitnik et al., Adv. Space Res., 32, 473 (2003)

[15] J.-P. Wuelser et al., Proc. SPIE, 5171, 111 (2004)

[16] J.R. Lemen et al., Sol. Phys. 275, 17 (2012)

[17] J.-P. Halain et al., Proc. SPIE, 7732, 20 (2010)

[18] E. Meltchakov et al., Appl. Phys. A, 98, 111 (2010)

[19] E. Meltchakov et al., Proc. SPIE, 8168, 59 (2011)

[20] F. Auchère et al., Proc. SPIE , 5901, 298 (2005)

[21] J. Gautier et al., Opt. Comm. 281(11), 3032 (2008)

[22] C. Hecquet et al., Appl. Phys. A, 95, 401 (2009) 